\title{
CDCA2 promotes tumorigenesis and induces radioresistance in oesophageal squamous cell carcinoma cells
}

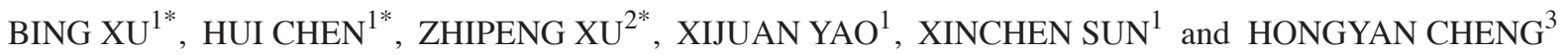 \\ ${ }^{1}$ Department of Radiation Oncology, The First Affiliated Hospital of Nanjing Medical University, Nanjing, Jiangsu 210029; \\ ${ }^{2}$ Department of Urology, Affiliated Zhongda Hospital of Southeast University, Nanjing, Jiangsu 210009; ${ }^{3}$ Department of \\ Synthetic Internal Medicine, The First Affiliated Hospital of Nanjing Medical University, Nanjing, Jiangsu 210029, P.R. China
}

Received October 11, 2020; Accepted April 15, 2021

DOI: $10.3892 / \mathrm{mmr} .2021 .12169$

\begin{abstract}
Cell division cycle-associated 2 (CDCA2) overexpression has been demonstrated to serve a significant role in tumorigenesis in certain types of cancer. Nevertheless, its role in tumour proliferation and radioresistance in oesophageal squamous cell carcinoma (ESCC) remains to be elucidated. Thus, the present study aimed to elucidate these roles. Data were downloaded from The Cancer Genome Atlas (TCGA) to compare the gene expression profiles. The expression of CDCA2 was higher in ESCC tissues compared with normal tissues. Gene set enrichment analysis was performed based on the ESCC cohorts in TCGA database. This demonstrated that higher expression of CDCA2 was significantly associated with the expression of related components of the cell cycle phase transition and $\mathrm{G}_{2} / \mathrm{M}$ phase transition pathways. Collectively, the results revealed that CDCA2 could serve as an underlying target to regulate tumour growth and radioresistance among patients with ESCC.
\end{abstract}

\section{Introduction}

Oesophageal cancer (EC) is one of the commonest types of cancer worldwide with high morbidity, especially in Eastern Asia (1-3). It is also the sixth leading cause of cancer-related death globally, with $5.3 \%$ of all cancer deaths

Correspondence to: Professor Hongyan Cheng, Department of Synthetic Internal Medicine, The First Affiliated Hospital of Nanjing Medical University, 300 Guangzhou Road, Nanjing, Jiangsu 210029, P.R. China

E-mail: chenghongyan68@126.com

Professor Xinchen Sun, Department of Radiation Oncology, The First Affiliated Hospital of Nanjing Medical University, 300 Guangzhou Road, Nanjing, Jiangsu 210029, P.R. China

E-mail: sunxinchen@njmu.edu.cn

*Contributed equally

Key words: tumorigenesis, radiosensitivity, The Cancer Genome Atlas, DNA repair, gene set enrichment analysis, cell cycle phase (age-standardized rates, 5.5 per 100,000) (2). EC has two main histological subtypes: Oesophageal adenocarcinoma (EA) and oesophageal squamous cell carcinoma (ESCC) (4). ESCC is one of the most aggressive squamous cell carcinomas and has a high prevalence in Asia, especially in China (5-7). Patients with advanced oesophageal squamous cell carcinoma who cannot tolerate or refuse to undergo surgery can choose radiotherapy (RT) $(4,7,8)$. As a number of patients may suffer radioresistance, the outcomes of clinical treatment are unsatisfactory (9). Hence, it is necessary to improve the clinical outcomes to explore the related molecular mechanisms of the proliferation and radioresistance of ESCC.

Cell division cycle-associated protein 2 (CDCA2; also known as RepoMan), regulates the phosphatase of the core substrates throughout the cell cycle (10). It has been reported that CDCA2 can promote cell proliferation in colorectal cancer by activating the AKT/cyclin D1 pathway (11). CDCA2, which is highly expressed in oral squamous cell carcinoma and lung adenocarcinoma, respectively, can promote the growth of certain types of tumour $(12,13)$. Previous studies have also reported that CDCA2 can modulate chromatin remodelling and DNA damage checkpoint activation $(14,15)$.

At present, the biological functions of CDCA2 in ESCC have rarely been reported and hence the purpose of the present study was to examine the expression and biological behaviours of CDCA2 and evaluate its role in the process of tumour growth and radioresistance in ESCC. Targeting CDCA2 may be a novel therapeutic option to heighten the radiosensitivity of ESCC.

\section{Materials and methods}

The Cancer Genome Atlas (TCGA) database. The expression data of all genes in ESCC were downloaded from TCGA, comprising 93 samples (11 normal tissues and 82 tumour tissues).

Cell culture. A total of five types of human ESCC cell lines (ECA109, KYSE150, KYSE450, TE10 and TE13) and an oesophageal epithelial cell line (SHEE) were maintained at the Jiangsu Province Hospital Core Facility Center. All cell lines involved in the present study were purchased from the Shanghai Institute of Biochemistry and Cell Biology. The cells were cultured in RPMI-1640 medium (Gibco; Thermo Fisher 
Scientific, Inc.) containing 10\% FBS (Gibco; Thermo Fisher Scientific, Inc.), $100 \mathrm{U} / \mathrm{ml}$ penicillin and $100 \mu \mathrm{g} / \mathrm{ml}$ streptomycin (Gibco; Thermo Fisher Scientific, Inc.). All cells were cultured in a humidified chamber with $5 \% \mathrm{CO}_{2}$ at $37^{\circ} \mathrm{C}$.

RNA extraction and reverse transcription-quantitative $(R T-q)$ $P C R$. In accordance with the manufacturer's instructions, total RNA was extracted from cells using TRIzol ${ }^{\circledR}$ (Thermo Fisher Scientific, Inc.). A NanoDrop spectrophotometer (ND-100, Thermo Fisher Scientific, Inc.) was used to detect the quality and concentration of RNA. RNA reverse transcription was conducted with a New Poly (A) Tailing kit (Thermo Fisher Scientific, Inc.) and a PrimeScript RT Master Mix kit (cat.no. RR036A; Takara Bio, Inc.). The temperature and duration of RT were as follows: $37^{\circ} \mathrm{C}$ for $15 \mathrm{~min}$, followed by $85^{\circ} \mathrm{C}$ for $5 \mathrm{sec}$ and $4^{\circ} \mathrm{C}$ for $10 \mathrm{~min}$. RT-PCR was performed utilizing Universal SYBR Green Master Mix (cat. no. 4913914001; Roche Diagnostics) with a 7500 Real-Time PCR System (Applied Biosystems; Thermo Fisher Scientific, Inc.). The thermocycling conditions were: $95^{\circ} \mathrm{C}$ for $10 \mathrm{~min}$, followed by 40 cycles at $95^{\circ} \mathrm{C}$ for $5 \mathrm{sec}, 55^{\circ} \mathrm{C}$ for $30 \mathrm{sec}$ and $72^{\circ} \mathrm{C}$ for $30 \mathrm{sec}$. Expression levels were calculated using the $2^{-\Delta \Delta \mathrm{Cq}}$ method (16). The relative mRNA expression was normalized to $\beta$-actin. All-in-one ${ }^{\mathrm{TM}}$ qPCR primers for CDCA2 were purchased from GeneCopoeia, Inc. All experiments were repeated three times. Primer sequences for $\mathrm{CDCA} 2$ and $\beta$-actin are as follows: CDCA2 forward, 5'-TGCCGAATTACCTCCTAATCCT-3' and reverse, 5'-TGCTCTACGGTTACTGTGGAAA-3'; and $\beta$-actin forward, 5'-CTCCATCCTGGCCTCGCTGT-3' and reverse, 5'-GCTGTCACCTTCACCGTTCC-3'.

Lentivirus transfection. Human CDCA2-targeting short hairpin (sh)RNA sequences (CCGGCTGTGGCAAGA GGGAAAGTAACTCGAGTTACTTTCCCTCTTGCCACA GTTTTTG) were cloned into hU6-MCS-CMW-puromycin (Shanghai GenePharma Co., Ltd.). The titre of lentivirus was $2.37 \times 10^{8} \mathrm{TU} / \mathrm{ml}$, and the best transduction efficiency in the current study was at multiplicity of infection $=5$. Lentivirus transduction was conducted according to the manufacturer's instructions. Lentivirus was added into cells at room temperature. Cells were placed in an incubator at $37^{\circ} \mathrm{C}$ for $12 \mathrm{~h}$, and then the medium was changed. As a negative control (NC), a shRNA with a scrambled sequence was used. The transfected cells were selected by puromycin until stably transfected cells were obtained. The concentration of puromycin in medium was $3 \mu \mathrm{g} / \mathrm{ml}$. Transfection efficiency was assessed by western blot analysis and RT-qPCR. Subsequent experiments were performed at least $72 \mathrm{~h}$ after transfection.

Cell Counting Kit-8 (CCK-8) assay. Cells were seeded in triplicate into a 96 -well plate at a density of 3,000 cells per well. Following cell adherence, $100 \mu \mathrm{l}$ mixed solution (CCK-8 solution: RPMI-1640 medium=1:10) was added to each well according to the manufacturer's instructions at the indicated time points (days 1, 2, 3 and 4). Following a 2-h incubation period at $37^{\circ} \mathrm{C}$, absorbance was determined with a microplate reader (cat. no. ELx800; BioTek Instruments, Inc.).

5-Ethynyl-2'-deoxyuridine (EdU) incorporation assay. An EdU assay kit (Guangzhou RiboBio Co., Ltd.) was used to assess cellular proliferation. Cells were cultured in RPMI-1640 medium containing $10 \% \mathrm{FBS}$ at $37^{\circ} \mathrm{C}$ in 24 -well plates in triplicate at a density of $5 \times 10^{4}$ cells per well overnight. Cells were then maintained for $2 \mathrm{~h}$ at $37^{\circ} \mathrm{C}$ in medium containing $50 \mu \mathrm{M}$ EdU and treated according to the manufacturer's instructions. Typical images were captured under a fluorescence microscope (magnification, $\mathrm{x} 40$; Nikon Corporation). The proportion of EdU-positive cells among the cells from three random fields was analysed using ImageJ software (Java 1.6.0_20; National Institutes of Health).

Clonogenic survival assays. Unequal numbers of exponentially growing cells ( 0 Gy: 300 cells, 2 Gy: 600 cells, 4 Gy: 1,200 cells, $6 \mathrm{~Gy}: 3,000$ cells and $8 \mathrm{~Gy}: 6,000$ cells) were plated into 6-well plates. Following cell adherence, they were subjected to X-ray radiation. Immediately after radiation, the culture medium (RPMI-1640 medium containing 10\% FBS) was renewed and the cells were cultured at $37^{\circ} \mathrm{C}$ for $\leq 12$ days. The cells were fixed and then stained with crystal violet at room temperature for $30 \mathrm{~min}$. The number of colonies (>50 cells/colony) were counted under a light microscope (magnification, x10). Plating efficiencies (PEs) were calculated as the number of colonies divided by the number of cells seeded. The surviving fraction (SF) of each radiation group was corrected by the PE of the nonradiated control. Dose-response clonogenic survival curves were plotted on a log-linear scale. Cell survival curves based on the mean survival fractions of the cell line were fitted to a multitarget single-hit model: $S=1-\left(1-\mathrm{e}^{-\mathrm{D} / \mathrm{D} 0}\right)^{\mathrm{N}}(17)$. The experiment was repeated three times.

Immunofluorescence. Immunofluorescence detection of $\gamma \mathrm{H} 2 \mathrm{AX}$ foci was utilized to evaluate DNA double-strand breaks (DSBs) in ESCC cells. The cells were seeded on a glass-bottomed dish and then irradiated with a single $8 \mathrm{~Gy}$ dose. Then, 2, 6 and $24 \mathrm{~h}$ after radiation, the cells were washed with PBS and fixed with $4 \%$ paraformaldehyde for $20 \mathrm{~min}$ at room temperature. Cells were stained with a rabbit anti- $\gamma \mathrm{H} 2 \mathrm{AX}$ monoclonal antibody (1:200; cat. no. ab229914; Abcam) overnight at $4^{\circ} \mathrm{C}$ and then with Alexa 555 Fluor secondary antibody (1:500; cat. no. A0453; Beyotime Institute of Biotechnology) for $1.5 \mathrm{~h}$ at room temperature. The nuclei were counterstained with $2 \mu \mathrm{g} / \mathrm{ml}$ DAPI (cat. no. C1005; Beyotime Institute of Biotechnology) for confocal microscopy(Zeiss AG; magnification, $\mathrm{x} 40$ ) for $30 \mathrm{~min}$ at room temperature.

Cell cycle detection. First, cells were seeded into a 6-well plate at a density of $1 \times 10^{5}$ cells per well and treated with 6-MV X-ray radiation at doses of 0 or $8 \mathrm{~Gy}$. Then, the cells were collected and stained with PI/RNase Staining Buffer (BD Biosciences) for $20 \mathrm{~min}$ at room temperature, according to the manufacturer's protocol. The cell cycle was detected using flow cytometry (FACSCalibur; BD Biosciences) and interpreted using FlowJo software (V10; FlowJo LLC). The experiment was repeated three times.

Western blotting. Total protein was separated from cell lysates on ice using RIPA buffer (Beyotime Institute of Biotechnology). An equal amount of protein $(40 \mu \mathrm{g})$, whose concentration was quantified by a BCA kit (Beyotime Institute of Biotechnology), was separated on a $10 \%$ SDS-polyacrylamide gel and then 

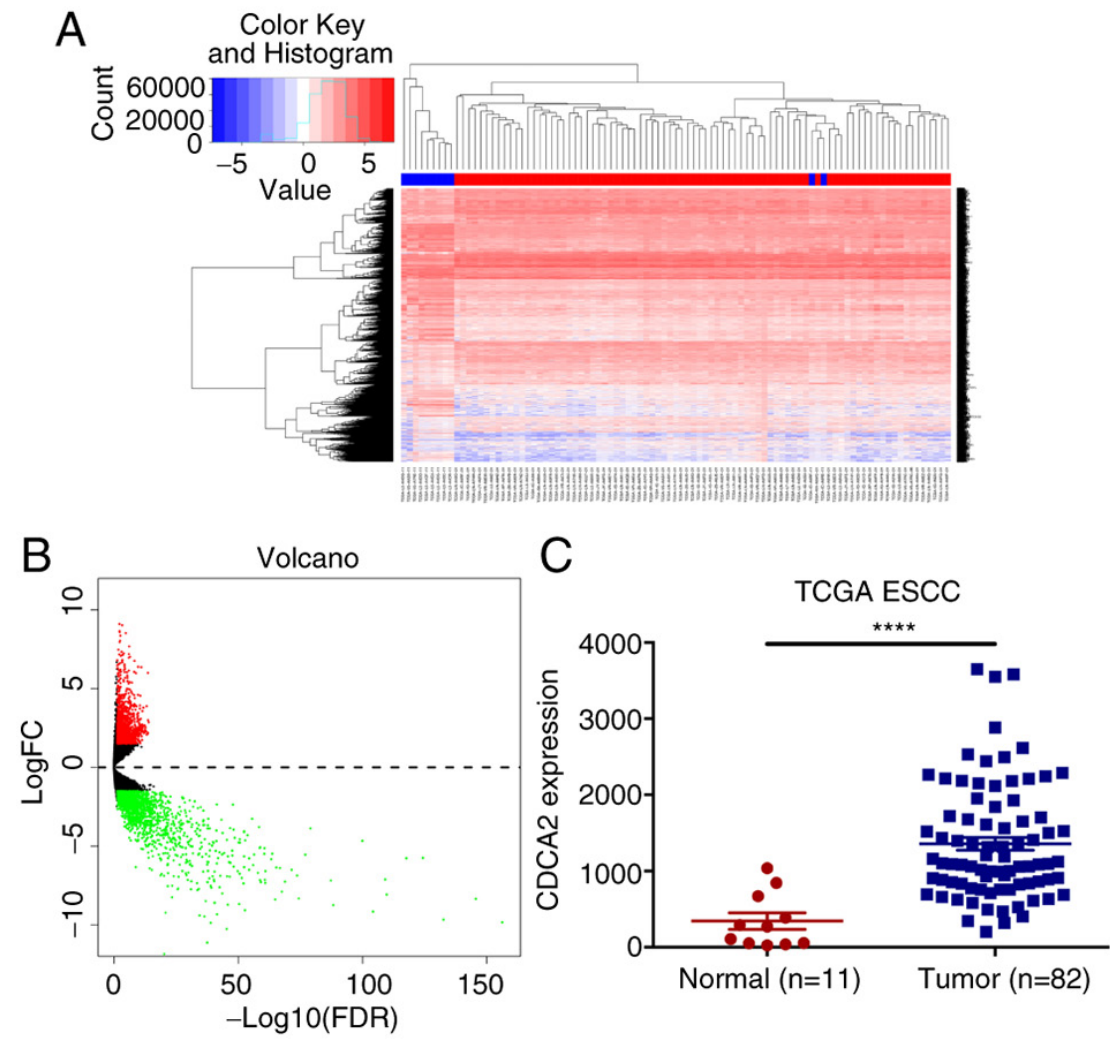

Figure 1. The expression of CDCA2 is upregulated in ESCC tissues. (A) Heatmap of the gene expression profiles of ESCC tissues and normal tissues basing on the data downloaded from TCGA. (B) Volcano map of the ESCC-related data extracted from TCGA. (C) The expression of CDCA2 in ESCC samples was significantly upregulated. ${ }^{* * * * *} \mathrm{P}<0.0001$. CDCA2, cell division cycle-associated 2; ESCC, oesophageal squamous cell carcinoma; TCGA, The Cancer Genome Atlas; FC, fold change; FDR, false discovery rate.

transferred to PVDF membranes. Afterwards, the membranes were blocked with $5 \%$ skimmed milk powder for $2 \mathrm{~h}$ at room temperature, followed by incubation with primary antibodies in dilution buffer at $4^{\circ} \mathrm{C}$ overnight. The next day, HRP-linked anti-rabbit secondary antibodies (1:3,000; cat. no. 7074; Cell Signaling Technology, Inc.) were incubated at room temperature for $2 \mathrm{~h}$. Western blotting analysis was conducted using a rabbit anti-CDCA2 monoclonal antibody (1:1,000; cat. no. 14976; Cell Signaling Technology, Inc.). An anti-GAPDH polyclonal antibody (1:5,000; cat. no. 10494-1-AP; ProteinTech Group, Inc.) was used as a loading control. The signals were visualized via an enhanced chemiluminescence detection kit (Thermo Fisher Scientific, Inc.) and a chemiluminescence detection system.

Gene Set Enrichment Analysis (GSEA). GSEA was performed using the Kyoto Encyclopaedia of Genes and Genomes (KEGG) pathway gene sets in the Molecular Signatures Database against two probe-level expression matrices via the GSEA v3.0 software (www.broadinstitute.org/gsea).

Xenograft tumours in nude mice. The present study was approved by the Institutional AnimalCare and Use Committee of Nanjing Medical University (approval no. 2103063). In total, 24 male BALB/c nude mice (17-20 g; 4-6 weeks old) were obtained from the Nanjing Medical University Animal Center and raised in a specific pathogen-free environment under a 12 -h light-dark cycle at $23 \pm 1^{\circ} \mathrm{C}$ and $50 \pm 5 \%$ humidity atmosphere. The mice were divided into four groups $(n=6)$ : i) shNC; ii) shCDCA2; iii) shNC and irradiation; and iv) shCDCA 2 and irradiation. A total of $2 \times 10^{6} \mathrm{KYSE} 450$ cells were subcutaneously implanted into the flanks of mice. At 20 days post injection, mice were exposed to irradiation (8 Gy) once in an RS 2000 Pro XRay Bioirradiator (Radsource) and then sacrificed at day 35. The lead shields were used to protect nontumor tissue from radiation damage. The mice were sacrificed with an intraperitoneal injection of $1 \%$ pentobarbital sodium at $100 \mathrm{mg} / \mathrm{kg}$. The criteria for death were sustained non-spontaneous breathing for 2-3 min and no blink reflex.

Statistical analysis. The data are expressed as the mean \pm standard deviation. Differences between groups were analysed using unpaired Student's t-test or one-way and mixed design ANOVA followed by Tukey's post hoc test. $\mathrm{P}<0.05$ was considered to indicate a statistically significant difference. Statistical analyses were performed using GraphPad Prism software (version 5.0; GraphPad Software, Inc.). All experiments were repeated at least three times.

\section{Results}

CDCA2 is upregulated in human ESCC tissues and cell lines. First, ESCC-related gene expression profiles of tumours and normal tissues were analysed using information extracted from TCGA database (Fig. 1A and B). The results showed 
A

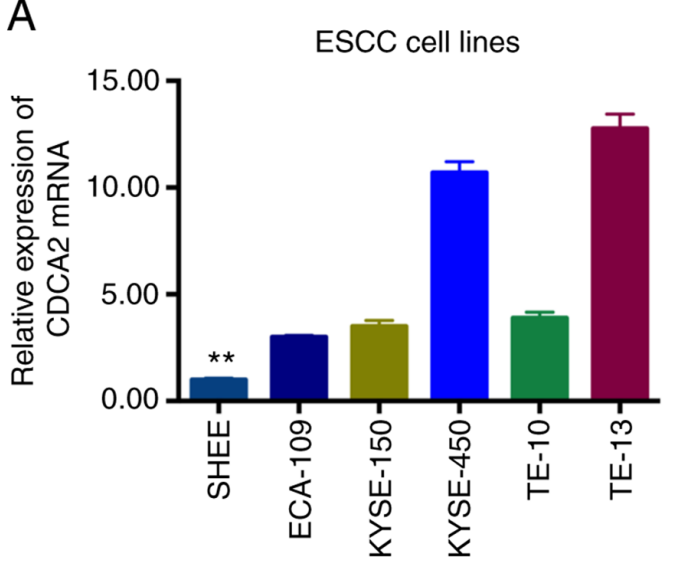

$\mathrm{D}$

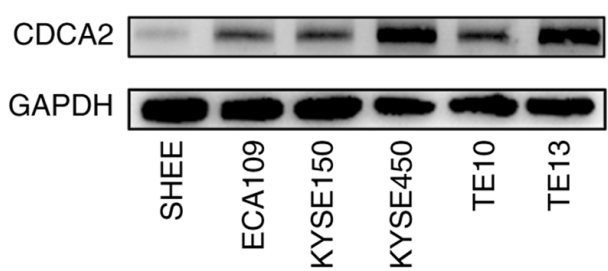

B

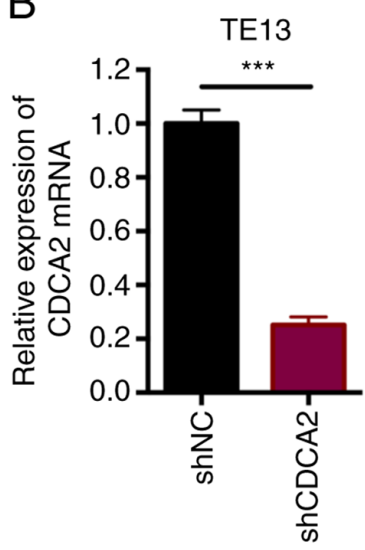

$E$

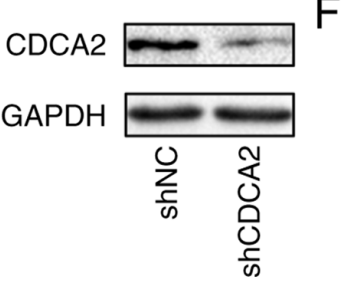

C
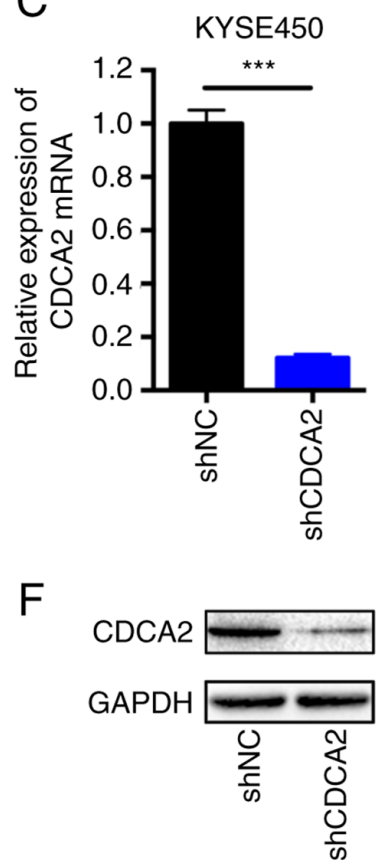

Figure 2. The expression of CDCA2 is upregulated in ESCC cell lines. (A) CDCA2 expression levels in the ESCC cell lines were detected by RT-qPCR. Mean \pm standard deviation, $n=3,{ }^{* *} \mathrm{P}<0.01$. (B and $\mathrm{C}$ ) Knockdown efficiency was verified by RT-qPCR in cells transfected with shCDCA2 lentivirus. Mean \pm standard deviation, $\mathrm{n}=3,{ }^{* * *} \mathrm{P}<0.001$. (D) CDCA2 expression levels in the ESCC cell lines as detected by western blot analysis. (E and F) Western blot analysis validated the transfection efficiency in cells transfected with shCDCA2 lentivirus. CDCA2, cell division cycle-associated 2; ESCC, oesophageal squamous cell carcinoma; RT-qPCR, reverse transcription-quantitative PCR; sh, short hairpin.

that, compared with the expression in normal tissues, the expression of CDCA2 in tumour samples was significantly upregulated (Fig. 1C). Afterwards, the CDCA2 expression levels were detected by RT-qPCR and western blot analysis in ESCC cell lines (Fig. 2A and D). As the results showed that CDCA2 was highly expressed in ESCC cells, especially in KYSE450 and TE13 cell lines, KYSE450 and TE13 cells were ultimately selected for further experiments. According to these results, it was hypothesized that CDCA2 might act as a regulator of tumour biological behaviour in ESCC. Since the function and mechanism of CDCA2 in the proliferation and radioresistance of ESCC cells remain unclear, it was intended to conduct related experiments.

To determine the important roles of CDCA2 in contributing to ESCC radioresistance, stable CDCA2 knockdown cell lines of both KYSE450 and TE13 cells were successfully generated by utilizing a specific shRNA against CDCA2. Then, RT-qPCR was performed to check the downregulation of CDCA2 in the two cell lines (Fig. 2B and C). Western blot analysis was also conducted to confirm the results (Fig. 2E and F).

CDCA2 promotes the proliferation of ESCC cells. CCK-8 assays were conducted to explore the influence of CDCA2 knockdown on ESCC proliferation. The proliferation rates of the cells in which the expression of CDCA2 was downregulated were significantly lower than those of the control groups (Fig. 3A and B). The results indicated that CDCA2 could regulate tumour growth and it was hypothesized that it might induce radioresistance in ESCC. The role of CDCA2 in cell proliferation was also verified via a more sensitive and specific EdU assay. As demonstrated in Fig. 3C and D,
CDCA2 depletion in KYSE450 cells reduced the number of EdU-positive cells. Similar results were observed in TE13 cells (Fig. 3E and F). According to these results, the downregulation of CDCA2 could effectively inhibit ESCC cell proliferation.

CDCA2 regulates the radiosensitivity of ESCC cells. The role of CDCA 2 in the radioresistance of ESCC was further determine. First, clonogenic survival assays were performed to assess the influence of CDCA2 on radiosensitivity. As demonstrated in Fig. 4A, the surviving cell fraction of KYSE450 cells with CDCA2 downregulation was distinctly lower compared with the control group, which was exposed to X-ray radiation. TE13 cells with reduced CDCA2 expression showed a similar outcome (Fig. 4B).

Immunofluorescence detection of $\gamma$-H2AX foci in KYSE450 and TE13 cell lines following X-ray radiation was used to explore whether CDCA2 was able to regulate radiation-induced DNA DSBs. As demonstrated in Fig. 4C-F, there were increased $\gamma-\mathrm{H} 2 \mathrm{AX}$ signals at different time points (2 and $24 \mathrm{~h}$ ) after irradiation in CDCA2-depleted cells compared with the control group. This suggested that downregulation of CDCA2 in ESCC cells could induce more DNA DSBs during radiation exposure. Collectively, downregulation of CDCA2 expression in oesophageal cancer cells enhanced cell radiation sensitivity.

CDCA2 influences the cell cycle distribution of ESCC cells. Previous studies have demonstrated that $\mathrm{G}_{2} / \mathrm{M}$ arrest is a pivotal mechanism for regulating radioresistance in ESCC (18-21). To explore whether CDCA2 could influence radiosensitivity in ESCC cells by regulating cell cycle distribution, every phase 
A

KYSE450

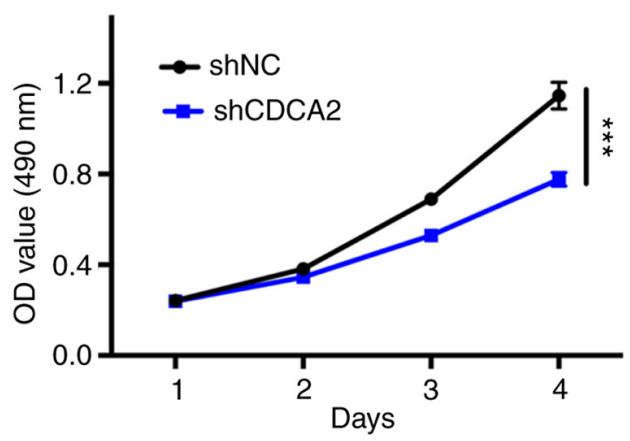

C

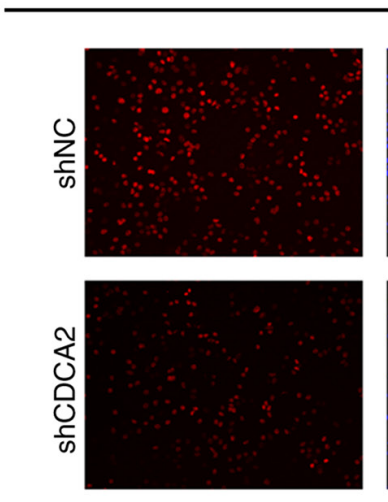

E
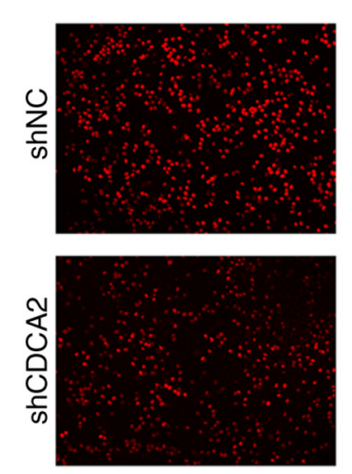

KYSE450
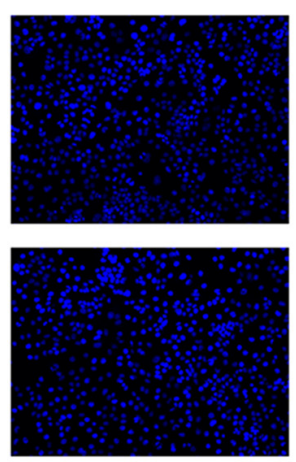

TE13
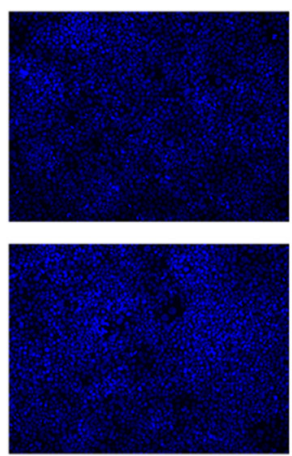

B

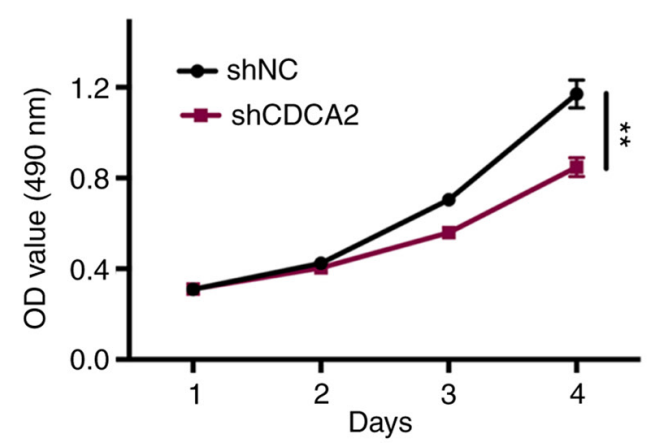

D

Figure 3. The knockdown of CDCA2 suppresses cell proliferation. CCK-8 assays were conducted to measure the rate of ESCC cell proliferation in (A) KYSE450/shCDCA2 and (B) TE13/shCDCA2 cells compared to that of the control groups. Mean \pm standard deviation, $\mathrm{n}=3,{ }^{* * *} \mathrm{P}<0.01,{ }^{* * * *} \mathrm{P}<0.001$. Typical images of the EdU incorporation assays and mean percentage of EdU positive cells in (C and D) KYSE450 cells and (E and F) TE13 cells with CDCA2 knockdown compared with the controls (blue fluorescence, cell nuclei; red fluorescence, EdU-positive cells) (magnification, x40). Mean \pm standard deviation, $\mathrm{n}=3,{ }^{* *} \mathrm{P}<0.01,{ }^{* * *} \mathrm{P}<0.001$. CDCA2, cell division cycle-associated 2; ESCC, oesophageal squamous cell carcinoma; sh, short hairpin; OD, optical density; sh, short hairpin; NC, negative control; EdU, 5-Ethynyl-2'-deoxyuridine.

percentage with CDCA2 knockdown after radiation was quantified (Fig. 5A-D). When exposed to X-ray radiation (8 Gy), the CDCA2-downregulated cells had a higher $\mathrm{G}_{2} / \mathrm{M}$ phase percentage than the controls in KYSE450 and TE13 cell lines (Fig. 5E and F).

To study the possible link between CDCA2 and the cell cycle phase transition pathway, GSEA was performed using the KEGG pathway gene sets in the Molecular Signatures Database against two probe-level expression matrices via the GSEA v3.0 software (www.broadinstitute.org/gsea). It was found that higher expression of CDCA2 was significantly associated with the expression of related components of the cell cycle phase transition and $\mathrm{G}_{2} / \mathrm{M}$ phase transition pathways (Fig. 6A and B). Collectively, the results revealed that CDCA 2 could regulate $G_{2} / M$ phase arrest to further influence radioresistance in ESCC cells.

CDCA2 influences ESCC cell proliferation and radiosensitivity in vivo. To confirm the effects of CDCA2 on ESCC cell proliferation in vivo, BALB/c nude mice were injected with TE13 cells transfected with shNC and shCDCA2. The tumour weight of shCDCA2 group was significantly lower compared 
A

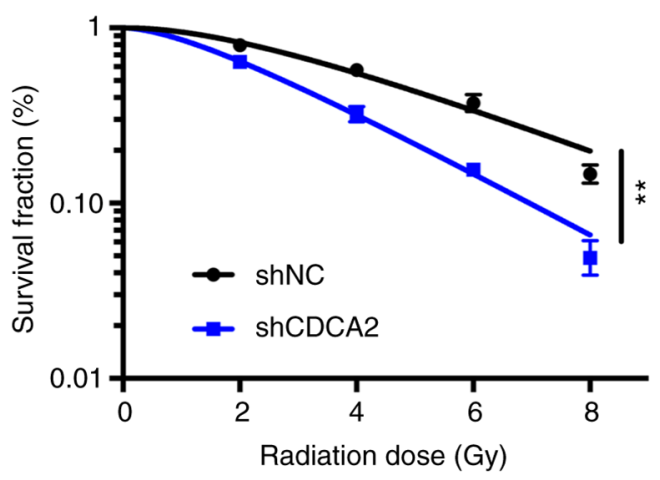

B

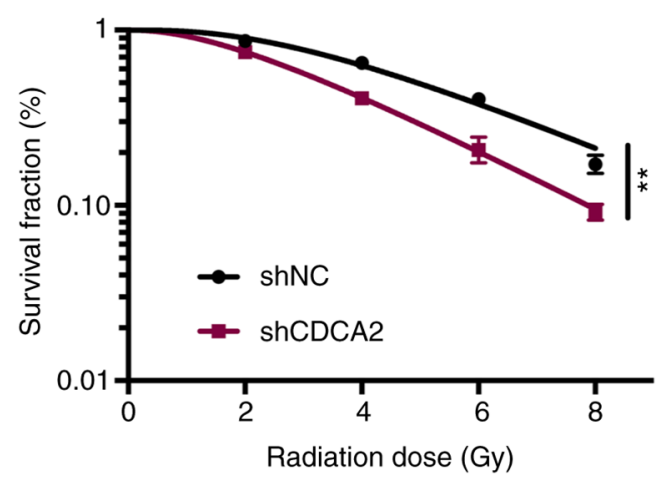

C

ShNC KYSE450
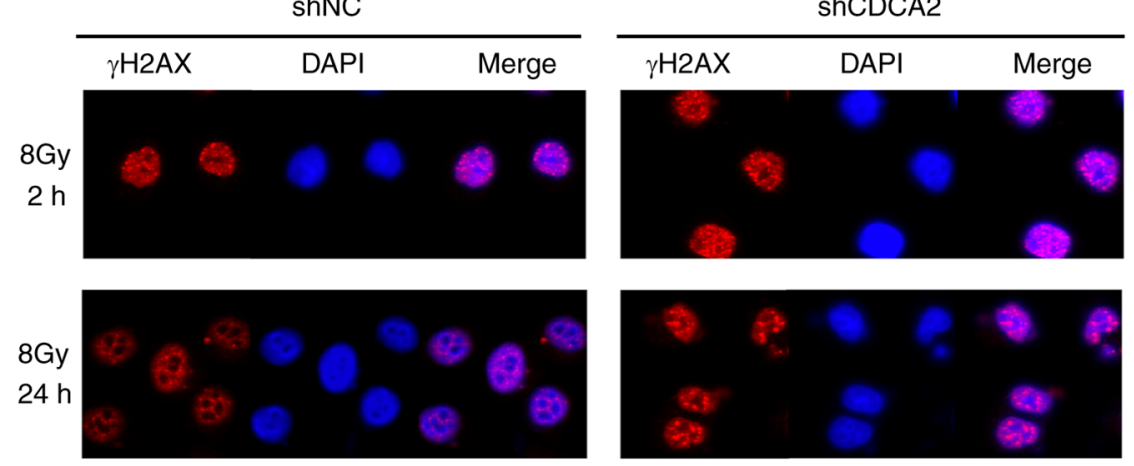

D
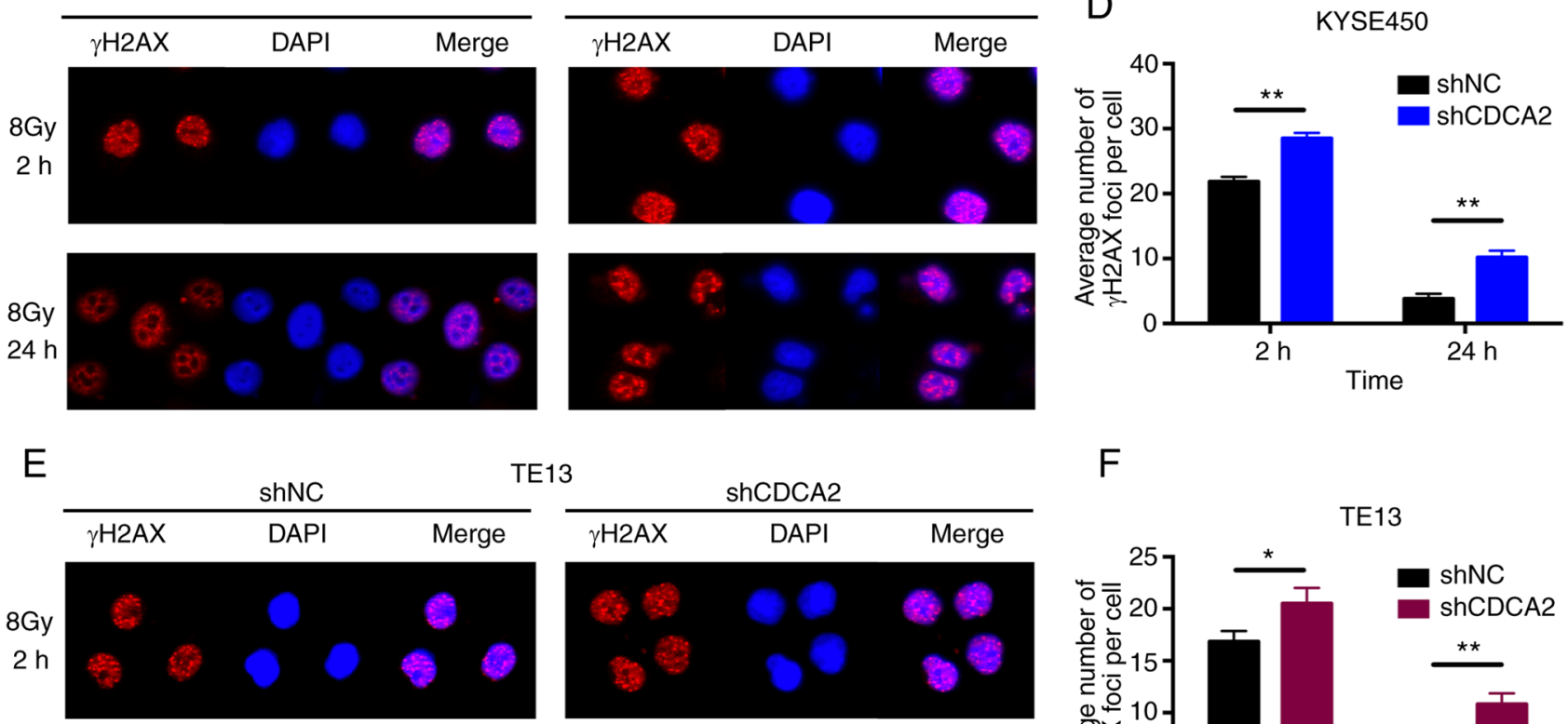

TE13

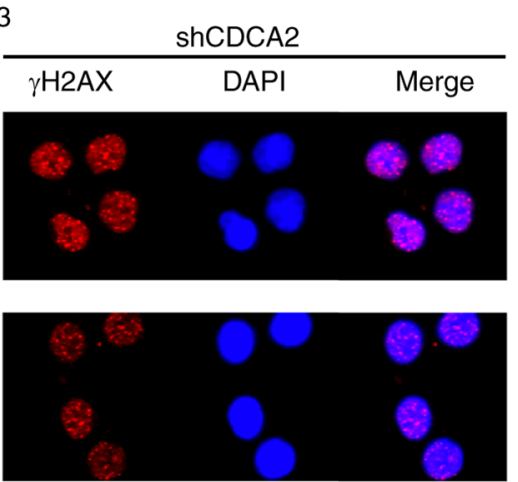

$\mathrm{F}$ TE13

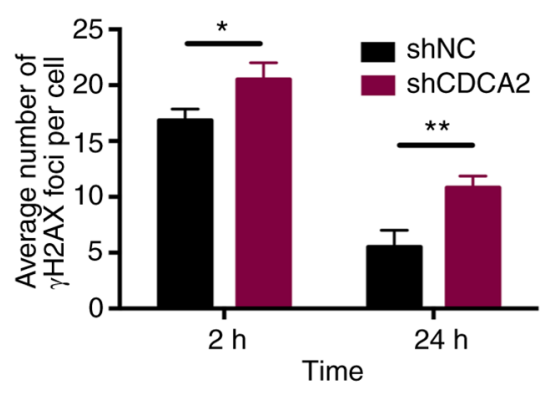

Figure 4. CDCA2 could regulate radiosensitivity in ESCC cells. The clonogenic survival assays was performed to compare the (A) KYSE450/shCDCA2 and (B) TE13/shCDCA2 cells to the control groups. The survival curves were calculated and fitted to a multi-target single-hit model. Mean \pm standard deviation, $\mathrm{n}=3,{ }^{* *} \mathrm{P}<0.01$. (C and E) Immunofluorescence staining of $\gamma \mathrm{H} 2 \mathrm{AX}$. (D and $\mathrm{F}$ ) The mean numbers of $\gamma \mathrm{H} 2 \mathrm{AX}$ foci were employed to evaluate radiation-induced double-strand break levels in ESCC cells (results from $\mathrm{C}$ and $\mathrm{E}$ ). Mean \pm standard deviation, $\mathrm{n}=3,{ }^{*} \mathrm{P}<0.05,{ }^{* *} \mathrm{P}<0.01$. CDCA2, cell division cycle-associated 2; ESCC, oesophageal squamous cell carcinoma; sh, short hairpin.

with control group. This effect was evident after exposure to radiation (Fig. 6C and D) and markedly evident following exposure to radiation. The in vivo results were consistent with the in vitro results. This further confirmed that downregulation of CDCA in ESCC cells can inhibit cell proliferation and improve tumour radiation sensitivity.

\section{Discussion}

Numerous studies have demonstrated that the differences in the expression of various genes between cancer and adjacent normal tissues led to innovations in diagnostic techniques and chemotherapy treatment strategies for cancer $(22,23)$. However, RT is regarded as the standard treatment modality in patients with ESCC who cannot be radically resected or those who refuse surgery (i.e., not fit for surgery) $(4,7,8)$. RT serves a vital role in the local control of ESCC4 (1). However, distant metastasis and local recurrence frequently occur, thus causing RT resistance $(1,9)$. Therefore, it is necessary to carry out genetic studies of oesophageal cancer. The expression levels of all known genes in oesophageal cancer and adjacent tissues were analysed in TCGA database. The present study confirmed that the expression of CDCA2 was higher in ESCC tissues compared with normal tissues. Several studies have demonstrated that CDCA2, as a cell division cycle-associated protein, is associated with tumour occurrence, progression and proliferation in several types of cancer, including melanoma, colorectal cancer, neuroblastoma, squamous cell carcinoma 


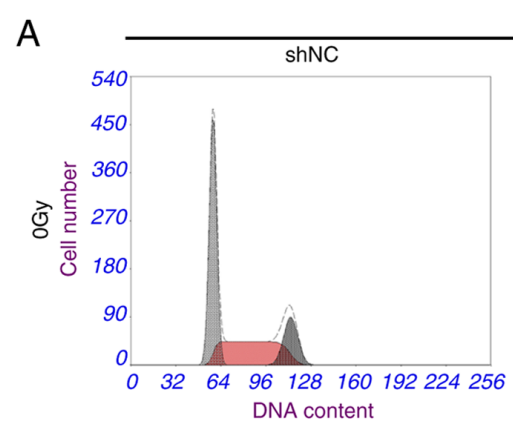

KYSE450
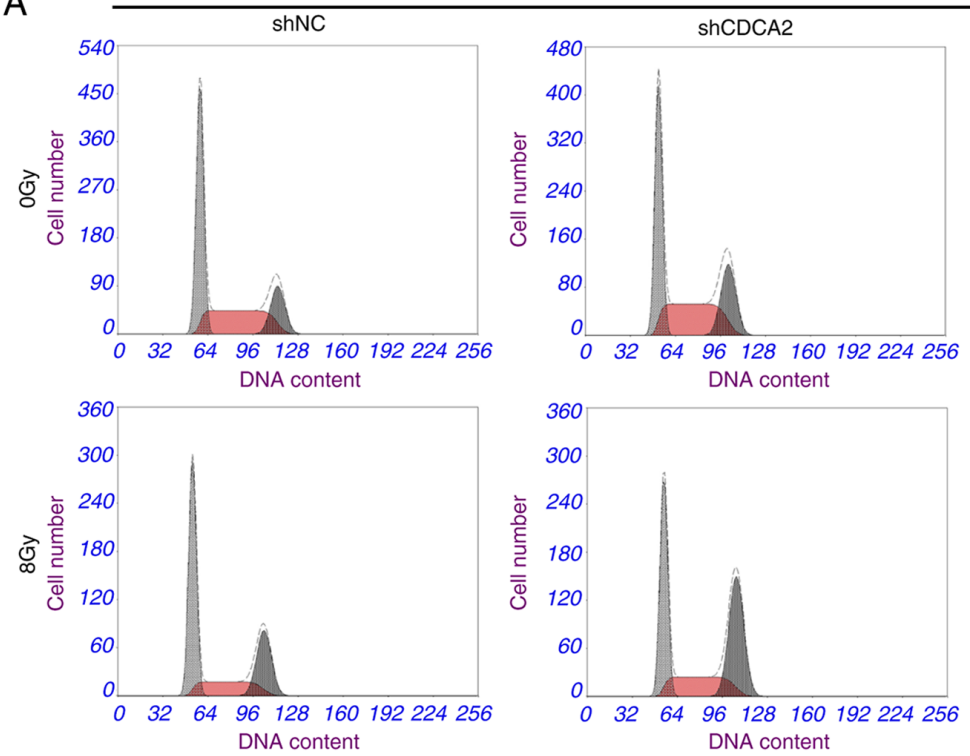

B
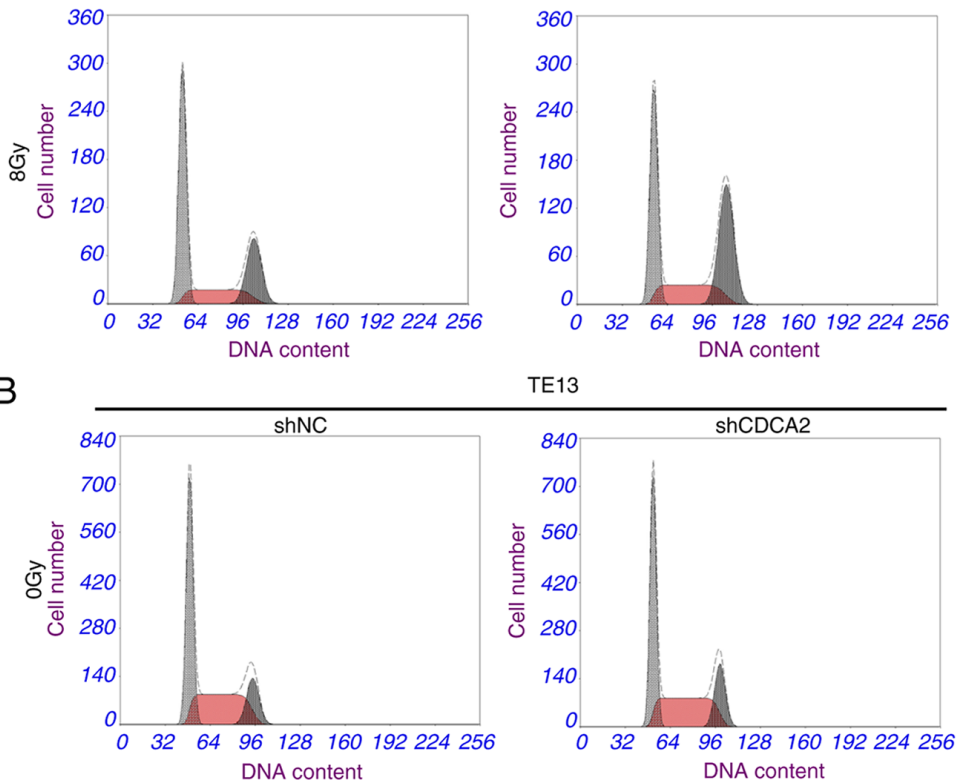

TE13
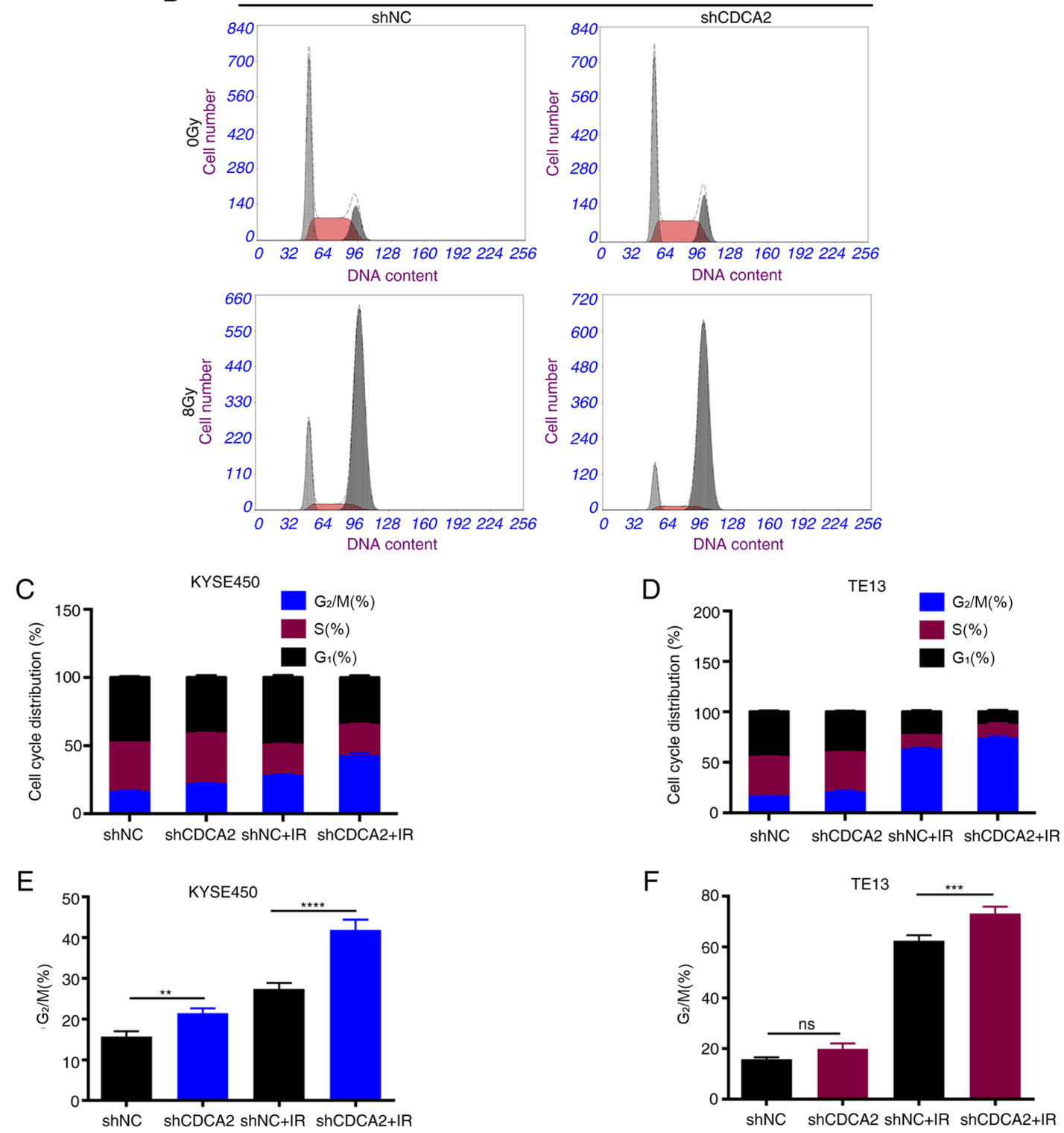

Figure 5. Inhibition of CDCA2 changes the cell cycle distribution in X ray-exposed ESCC cells. Cell cycle distribution of (A) KYSE450/shCDCA2 and (B) TE13/shCDCA2 cells. (C and D) Phase percentage with CDCA2 knockdown following radiation. (E and F) Downregulation of CDCA2 had a higher $\mathrm{G}_{2} / \mathrm{M}$ phase percentage. Mean \pm standard deviation, $\mathrm{n}=3,{ }^{* * *} \mathrm{P}<0.01,{ }^{* * * *} \mathrm{P}<0.001,{ }^{* * * * *} \mathrm{P}<0.0001$. CDCA2, cell division cycle-associated 2; ESCC, oesophageal squamous cell carcinoma; sh, short hairpin; NC, negative control; IR, ionising radiation; ns, not significant. 
A

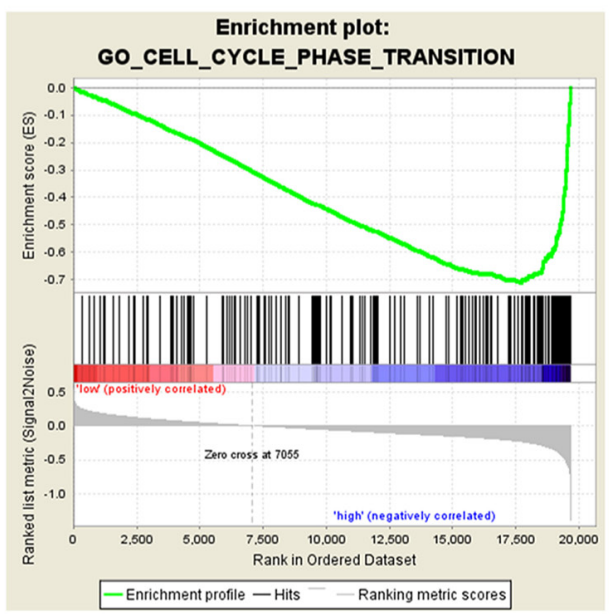

C

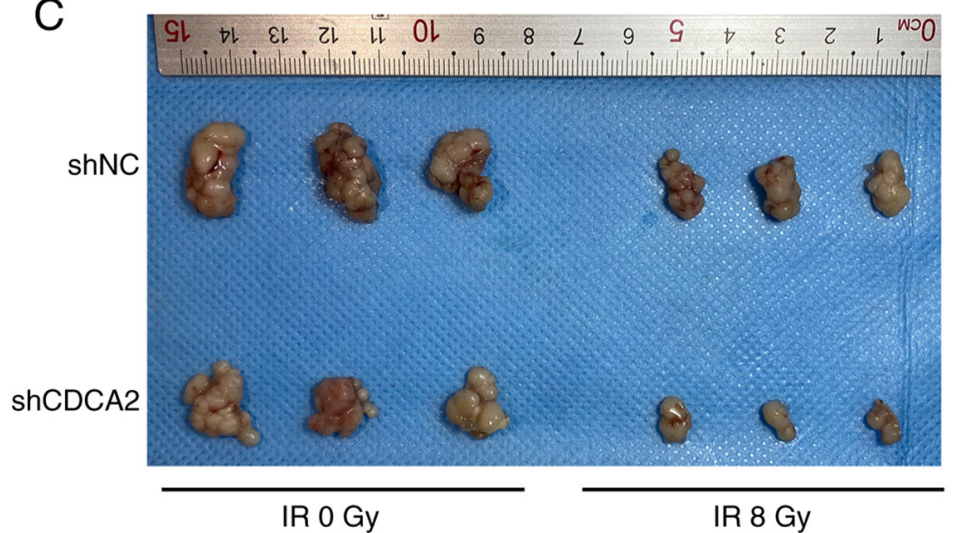

B

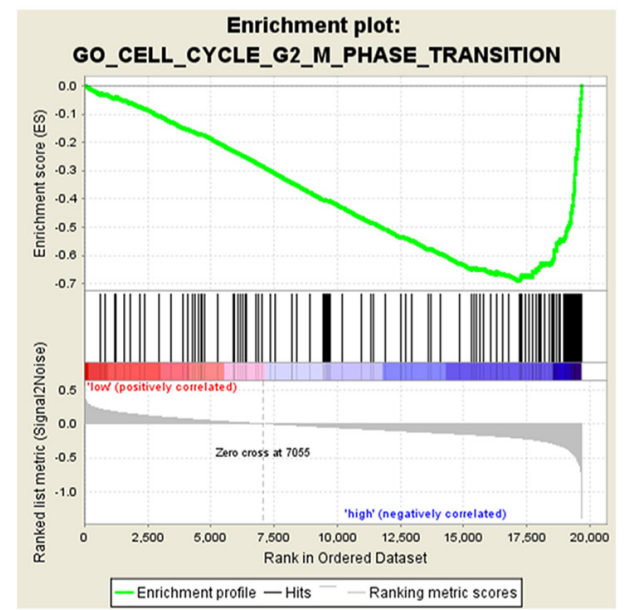

TE13

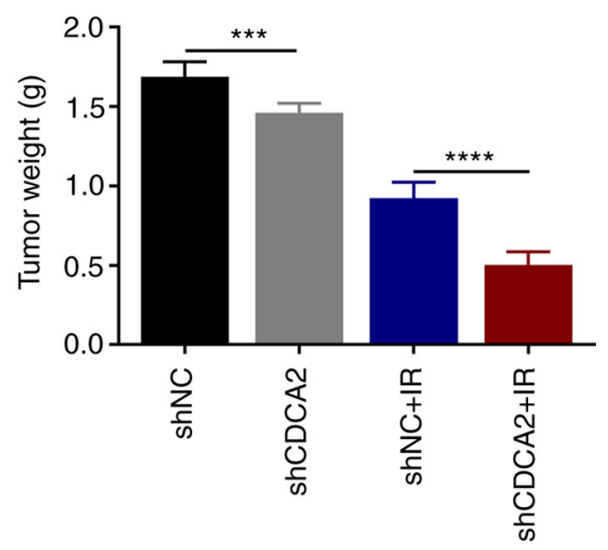

Figure 6. GSEA analysis of CDCA2. The knockdown of CDCA2 suppresses cell proliferation in vivo. (A and B) GSEA analysis showed that CDCA2 was associated with cell cycle phase transition pathways. (C) Representative xenografts each group were shown ( $\mathrm{n}=3$ ). (D) Tumour weight was recorded and presented as mean \pm standard deviation. ${ }^{* * *} \mathrm{P}<0.001,{ }^{* * * * *} \mathrm{P}<0.0001$. GSEA, gene set enrichment analysis; CDCA2, cell division cycle-associated $2 ;$ sh, short hairpin; NC, negative control; IR, ionising radiation.

and others $(11-13,24,25)$. Ionizing radiation affects cell proliferation through the cell cycle and cells at different stages of the cycle exhibit different radiation sensitivities $(26,27)$. Hence, the present study explored the association between CDCA2 and radioresistance. To date, no reports, to the best of the authors' knowledge have examined the role of CDCA2 in ESCC radioresistance to induce radiosensitivity.

The in vitro experiments validated the abovementioned hypothesis. CDCA2 knockdown decreased ESCC cell proliferation. Dose-dependent clone formation was used to explore whether CDCA2 can affect radiosensitivity and the downregulation of CDCA2 inhibited the formation of cloning. These results revealed the action of CDCA2 in the regulation of ESCC radiosensitivity. DNA is the main target of ionizing radiation (28). The efficacy of RT depends on its ability to induce DNA damage in cancer cells (29). The ability of a cell to repair DNA damage caused by radiation will finally affect whether it could succumb to cell death (30). The proficiency of DNA damage repair and DNA repair processes is associated with tumour resistance to ionizing radiation (29). Inhibition of DNA damage repair can cause cell cycle arrest or programmed cell death (31).
Histone $\mathrm{H} 2 \mathrm{AX}$ is phosphorylated near DNA double-strand breaks and $\gamma \mathrm{H} 2 \mathrm{AX}$ can be used as a marker for DNA DSBs in chromatin and can be used to assess radiosensitivity (32). $\gamma \mathrm{H} 2 \mathrm{AX}$ phosphorylation foci were significantly higher in shCDCA2 cells at 2 and $24 \mathrm{~h}$ after radiation compared with the control groups. The persistence of $\gamma \mathrm{H} 2 \mathrm{AX}$ lesions has previously been demonstrated to be associated with radiosensitivity and the loss of lesions depends on effective DNA repair. As DNA is the main target of ionizing radiation and that DNA DSBs are a key lesion that causes cell death, CDCA2 knockdown can increase ESCC sensitivity to X-ray radiation $(31,33)$.

Generally, cells are sensitive to radiation-induced DNA damage during $\mathrm{G}_{2} / \mathrm{M}$ and $\mathrm{G}_{1} / \mathrm{S}$, while cells in the late $S$ phase are most resistant to ionizing radiation (34). $\mathrm{G}_{2} / \mathrm{M}$ phase arrest is the most sensitive stage of cell damage. After cells were exposed to X-ray irradiation, CDCA2 knockdown increased radiation-induced $\mathrm{G}_{2} / \mathrm{M}$ phase arrest, as demonstrated by flow cytometry analysis.

Previous reports have demonstrated that downregulation of CDCA2 can induce $G_{1}$ arrest of lung adenocarcinoma cells and oral squamous cell carcinoma cells $(12,13)$. In the 
present study, CDCA2 showed a regulatory effect on the cycle distribution of oesophageal squamous cell carcinoma cells, however, it was the $\mathrm{G}_{2} / \mathrm{M}$ phase rather than the $\mathrm{G}_{1}$ phase. Radiation can increase the distribution in the $G_{2} / M$ phase and the present study showed that radiation combined with downregulation of CDCA 2 can aggravate this effect. The reason may be that CDCA2 did not serve the $\mathrm{G}_{1}$-arresting function in these two cell lines, or the sample size was too small. The results of the present study did not contradict previous studies. To explore the relationship between CDCA2 and the cell cycle, GSEA was also conducted using the data of the ESCC cohorts from the TCGA database. Please provide a reference for this statement, remembering to update the reference list and in-text citations accordingly. The results of the present study revealed that CDCA2 could regulate the cell cycle distribution to further influence radioresistance in ESCC cells. However, the findings and mechanisms in the present study should be further tested in animal models and patient samples.

In conclusion, the present study found that silencing CDCA 2 could suppress the growth and enhance the radiosensitivity of ESCC cells. CDCA2 is a potential molecular target of radiosensitization.

\section{Acknowledgements}

Not applicable.

\section{Funding}

The present study was supported by the National Natural Science Foundation of China [grant no. 81672983 (BA16)].

\section{Availability of data and materials}

The datasets used and/or analyzed during the current study are available from the corresponding author on reasonable request.

\section{Authors' contributions}

BX, HuC, HoC and XS conceived the study, BX and $\mathrm{HuC}$ participated in data collection, analysis and interpretation, and drafted the manuscript. ZX and XY contributed to collecting samples and materials, and analyzing data. BX, HoC, ZX and XY participated in the analysis and interpretation of the results. All authors read and approved the final manuscript, and consented to publish this manuscript. XS and HYC confirmed the authenticity of all the raw data.

\section{Ethics approval and consent to participate}

The present study was approved by the Institutional Animal Care and Use Committee of Nanjing Medical University (approval no. 2103063) and was in accordance with China's National Code of the Animal Care for Scientific Experimentation.

\section{Patient consent for publication}

Not applicable.

\section{Competing interests}

The authors declare that they have no competing interests.

\section{References}

1. Siegel RJ, Miller KD and Jemal A: Cancer statistics, 2019. CA Cancer J Clin 69: 7-34, 2019.

2. Bray F, Ferlay J, Soerjomataram I, Siegel RL, Torre LA and Jemal A: Global cancer statistics 2018: GLOBOCAN estimates of incidence and mortality worldwide for 36 cancers in 185 countries. CA Cancer J Clin 68: 394-424, 2018.

3. Ferlay J, Colombet M, Soerjomataram I, Mathers C, Parkin DM, Pineros M, Znaor A and Bray F: Estimating the global cancer incidence and mortality in 2018: GLOBOCAN sources and methods. Int J Cancer 144: 1941-1953, 2019.

4. Le Bras GF, Farooq MH, Falk GW and Andl CD: Esophageal cancer: The latest on chemoprevention and state of the art therapies. Pharmacol Res 113A: A236-A244, 2016.

5. Zhao J, He YT, Zheng RS, Zhang SW and Chen WQ: Analysis of esophageal cancer time trends in China, 1989-2008. Asian Pac J Cancer Prev 13: 4613-4617, 2012.

6. Ohashi S, Miyamoto S, Kikuchi O, Goto T, Amanuma Y and Muto M: Recent advances from basic and clinical studies of esophageal squamous cell carcinoma. Gastroenterology 149: 1700-1715, 2015.

7. Liang H, Fan JH and Qiao YL: Epidemiology, etiology, and prevention of esophageal squamous cell carcinoma in China. Cancer Biol Med 14: 33-41, 2017.

8. van Rossum PSN, Mohammad NH, Vleggaar FP and van Hillegersberg R: Treatment for unresectable or metastatic oesophageal cancer: Current evidence and trends. Nat Rev Gastroenterol Hepatol 15: 235-249, 2018.

9. Dai T and Shah MA: Chemoradiation in oesophageal cancer. Best Pract Res Clin Gastroenterol 29: 193-209, 2015.

10. Prevost M, Chamousset D, Nasa I, Freele E, Morrice N, Moorhead G and Trinkle-Mulcahy L: Quantitative fragmentome mapping reveals novel, domain-specific partners for the modular protein RepoMan (recruits PP1 onto mitotic chromatin at anaphase). Mol Cell Proteomics 12: 1468-1486, 2013.

11. Feng Y, Qian W, Zhang Y, Peng W, Li J, Gu Q, Ji D, Zhang Z, Wang Q, Zhang D and Sun Y: CDCA2 promotes the proliferation of colorectal cancer cells by activating the AKT/CCND1 pathway in vitro and in vivo. BMC Cancer 19: 576, 2019.

12. Uchida F, Uzawa K, Kasamatsu A, Takatori H, Sakamoto Y, Ogawara K, Shiiba M, Bukawa H and Tanzawa H: Overexpression of CDCA2 in human squamous cell carcinoma: Correlation with prevention of G1 phase arrest and apoptosis. PLoS One 8: e56381, 2013.

13. Shi R, Zhang C, Wu Y, Wang X, Sun Q, Sun J, Xia W, Dong G, Wang A, Jiang F and Xu L: CDCA2 promotes lung adenocarcinoma cell proliferation and predicts poor survival in lung adenocarcinoma patients. Oncotarget 8: 19768-19779, 2017.

14. Peng A, Lewellyn AL, Schiemann WP and Maller JL: Repo-man controls a protein phosphatase 1-dependent threshold for DNA damage checkpoint activation. Curr Biol 20: 387-396, 2010.

15. Vagnarelli P: Repo-man at the intersection of chromatin remodelling, DNA repair, nuclear envelope organization, and cancer progression. Adv Exp Med Biol 773: 401-414, 2014.

16. Livak KJ and Schmittgen TD: Analysis of relative gene expression data using real-time quantitative PCR and the 2(-Delta Delta C(T)) Method. Methods 25: 402-408, 2001.

17. Zhao Y, Yi J, Tao L, Huang G, Chu X, Song H and Chen L: Wnt signaling induces radioresistance through upregulating HMGB1 in esophageal squamous cell carcinoma. Cell Death Dis 9: 433, 2018.

18. Dillon MT, Good JS and Harrington KJ: Selective targeting of the $\mathrm{G} 2 / \mathrm{M}$ cell cycle checkpoint to improve the therapeutic index of radiotherapy. Clin Oncol (R Coll Radiol) 26: 257-265, 2014.

19. Sinclair WK and Morton RA: X-ray sensitivity during the cell generation cycle of cultured Chinese hamster cells. Radiat Res 29: 450-474, 1966.

20. Terasima T and Tolmach LJ: Variations in several responses of HeLa cells to $\mathrm{x}$-irradiation during the division cycle. Biophys J 3: 11-33, 1963.

21. Pawlik TM and Keyomarsi K: Role of cell cycle in mediating sensitivity to radiotherapy. Int J Radiat Oncol Biol Phys 59: 928-942, 2004. 
22. Zheng N, Zhang S, Wu W, Zhang N and Wang J: Regulatory mechanisms and therapeutic targeting of vasculogenic mimicry in hepatocellular carcinoma. Pharmacol Res 166: 105507, 2021.

23. Petersen EV, Chudakova DA, Skorova EY, Anikin V, Reshetov IV and Mynbaev OA: The extracellular matrix-derived biomarkers for diagnosis, prognosis, and personalized therapy of malignant tumors. Front Oncol 10: 575569, 2020.

24. Krasnoselsky AL, Whiteford CC, Wei JS, Bilke S, Westermann F, Chen QR and Khan J: Altered expression of cell cycle genes distinguishes aggressive neuroblastoma. Oncogene 24: $1533-1541,2005$

25. Xiao B, Chen L, Ke Y, Hang J, Cao L, Zhang R, Zhang W, Liao Y, Gao Y, Chen J, et al: Identification of methylation sites and signature genes with prognostic value for luminal breast cancer. BMC Cancer 18: 405, 2018.

26. Chang L, Graham PH, Hao J, Ni J, Bucci J, Cozzi PJ, Kearsley JH and Li Y: PI3K/Akt/mTOR pathway inhibitors enhance radiosensitivity in radioresistant prostate cancer cells through inducing apoptosis, reducing autophagy, suppressing NHEJ and HR repair pathways. Cell Death Dis 5: e1437, 2014.

27. He G, Di X, Yan J, Zhu C, Sun X and Zhang S: Silencing human epidermal growth factor receptor-3 radiosensitizes human luminal A breast cancer cells. Cancer Sci 109: 3774-3782, 2018.

28. Buckley AM, Lynam-Lennon N, O'Neill H and O'Sullivan J: Targeting hallmarks of cancer to enhance radiosensitivity in gastrointestinal cancers. Nat Rev Gastroenterol Hepatol 17: 298-313, 2020.
29. Sadoughi F, Mirsafaei L, Dana PM, Hallajzadeh J, Asemi Z, Mansournia MA, Montazer M, Hosseinpour M and Yousefi B: The role of DNA damage response in chemo- and radio-resistance of cancer cells: Can DDR inhibitors sole the problem? DNA Repair (Amst) 101: 103074, 2021.

30. Lynam-Lennon N, Reynolds JV, Pidgeon GP, Lysaght J, Marignol L and Maher SG: Alterations in DNA repair efficiency are involved in the radioresistance of esophageal adenocarcinoma. Radiat Res 174: 703-711, 2010.

31. Liu C, Gross N, Li Y, Li G, Wang Z, Zhong S, Li Y and Hu G: PARP inhibitor Olaparib increases the sensitization to radiotherapy in FaDu cells. J Cell Mol Med 24: 2444-2450, 2020.

32. Sak A and Stuschke M: Use of gammaH2AX and other biomarkers of double-strand breaks during radiotherapy. Semin Radiat Oncol 20: 223-231, 2010.

33. Bouzinab K, Summers HS, Stevens MFG, Moody CJ, Thomas NR, Gershkovich P, Weston N, Ashford MB, Bradshaw TD and Turyanska L: Delivery of temozolomide and N3-propargyl analog to brain tumors using an apoferritin nanocage. ACS Appl Mater Interfaces 12: 12609-12617, 2020.

34. Maity A, McKenna WG and Muschel RJ: The molecular basis for cell cycle delays following ionizing radiation: A review. Radiother Oncol 31: 1-13, 1994.

(i) (3) This work is licensed under a Creative Commons Attribution-NonCommercial-NoDerivatives 4.0 International (CC BY-NC-ND 4.0) License. 